\section{SELECTED GLOBAL HEALTH ASSESSMENT TOOLS SIGNIFICANTLY PREDICT POSTOPERATIVE CLINICAL OUTCOME IN ELDERLY PATIENTS WITH OVARIAN CANCER}

${ }^{1} \mathrm{~K}$ Anic ${ }^{*},{ }^{1} \mathrm{~F}$ Flohr, ${ }^{1} \mathrm{MW}$ Schmidt, ${ }^{1} \mathrm{~S}$ Krajnak, ${ }^{1} \mathrm{~V}$ Linz, ${ }^{1} \mathrm{R}$ Schwab, ${ }^{2} \mathrm{C}$ Westphalen, ${ }^{3}$ EK Hartmann, ${ }^{1} \mathrm{M}$ Schmidt, ${ }^{1} \mathrm{~A}$ Hasenburg, ${ }^{1} \mathrm{M}$ Battista. ${ }^{1}$ University Medical Centre of the Johannes Gutenberg University Mainz, Department of Gynaecology and Obstetrics, Mainz, GERMANY; ${ }^{2}$ University Medical Centre of the Johannes Gutenberg University Mainz, Department of Geriatric Medicine, Mainz, GERMANY; ${ }^{3}$ University Medical Centre of the Johannes Gutenberg University Mainz, Department of Anesthesiology, Mainz, GERMANY

\subsection{6/ijgc-2021-ESG0.364}

Introduction/Background* This study evaluated the impact of the global health status on the rate of postoperative clinical complications in patients with ovarian cancer (OC) older than 60 years.

Methodology Selected global health status assessment tools were retrospectively determined in a cohort of OC patients older than 60 years, treated at our institution between 2008 and 2019. The primary outcome was the incidence and type of postoperative clinical complications in accordance with the Veteran Affairs' National Surgical Quality Improvement Program (NSQIP). Differences in binary and ordinal variables between independent groups were analyzed by the chi-square test. Furthermore, rank correlations were examined with Spearman-Correlation.

Result(s)* 116 patients entered the study. 54 patients $(46.6 \%)$ were classified as G-8 frail and 56 patients (48.3\%) as G-8 non-frail. CCI classified 24 patients (20.7\%) into CCI-1, 63 patients (54.3\%) into CCI-2 and 29 patients (25.0\%) into CCI-3. The ASA PS allocated 48 patients (44.0\%) as ASA-2 and 61 patients $(56.0 \%)$ as ASA-3. The Lee-Index allocated 60 patients $(61.9 \%)$ to a cohort with a four-year-mortality $<20 \%$ and 37 patients (38.1\%) with a higher mortality. Two patients died within 60 days after surgery in the G-8 frail group and the CCI-2 cohort. In total, 23 patients (19.8\%) suffered from perioperative therapy relevant clinical complications (e.g. cardiopulmonary $(11.8 \%)$ or multiorgan complications $(6.9 \%))$. The total rates of relevant postoperative clinical complications were significantly higher in the group of G-8 frail patients $(13.6 \%$ vs. $6.4 \% ; \mathrm{p}=0.045)$ and the CCI- 2 and CCI-3 groups (CCI-2: 12.1\%, CCI-3: 6.9\% vs. CCI-1: 0.9\%; $\mathrm{p}=0.081)$. ASA PS, Lee-Index and age alone were not able to predict the clinical complications. Furthermore, G-8 frail and CCI-frail status were significantly associated with an administration of more perioperative transfusions (G-8: $32.7 \%$ vs. $15.3 \%$; $\mathrm{p}=0.031$ and CCI- $2+$ CCI- $3: 31.5 \%+16.2 \%$ vs. $3.6 \%$; $\mathrm{p}=0.002)$. Neither global health status nor age showed an association with postoperative revisions or length of hospitalization. Conclusion* Preoperative evaluation of global health status with G-8 score and CCI were significantly associated with elevated rates of postoperative complications and the need of transfusions, in contrast to age alone in elderly patients with OC.

\section{RISKS AND BENEFITS OF SYSTEMATIC LYMPHADENECTOMY DURING INTERVAL DEBULKING SURGERY FOR ADVANCED HIGH GRADE SEROUS OVARIAN CANCER}

L Benoit*, M Koual, MA Le Frere Belda, J Zerbib, L Fournier, HT Nguyen Xuan, N Delanoy, E Bentivegna, AS Bats, H Azaïs. European Hospital Georges Pompidou, Paris, France
Introduction/Background* Recent findings have suggested that lymphadenectomy during primary cytoreductive surgery (CRS) in patients with ovarian cancer was not associated with an improve in survival, at the expense of a high morbidity. The aim of our study was to evaluate the impact of lymphadenectomy in patients with high-grade serous ovarian cancer benefitting from neoadjuvant chemotherapy (NACT) followed by interval debulking surgery (IDS).

Methodology A retrospective, unicentric study including all patients undergoing NACT and IDS was carried out from 2005-2018. Patients with and without lymphadenectomy were compared in terms of recurrence free survival (RFS), overall survival (OS), and complication rates. Survival was assessed using a Cox regression model and Kaplan Meyer curves.

Result(s)* We included 203 patients. Of these, 133 had a lymphadenectomy $(65.5 \%)$ and 77 had involved nodes $(57.9 \%)$. Patients who did not benefit from a lymphadenectomy were older with a more extensive disease and lesser rate of complete CRS. No differences were noted between the
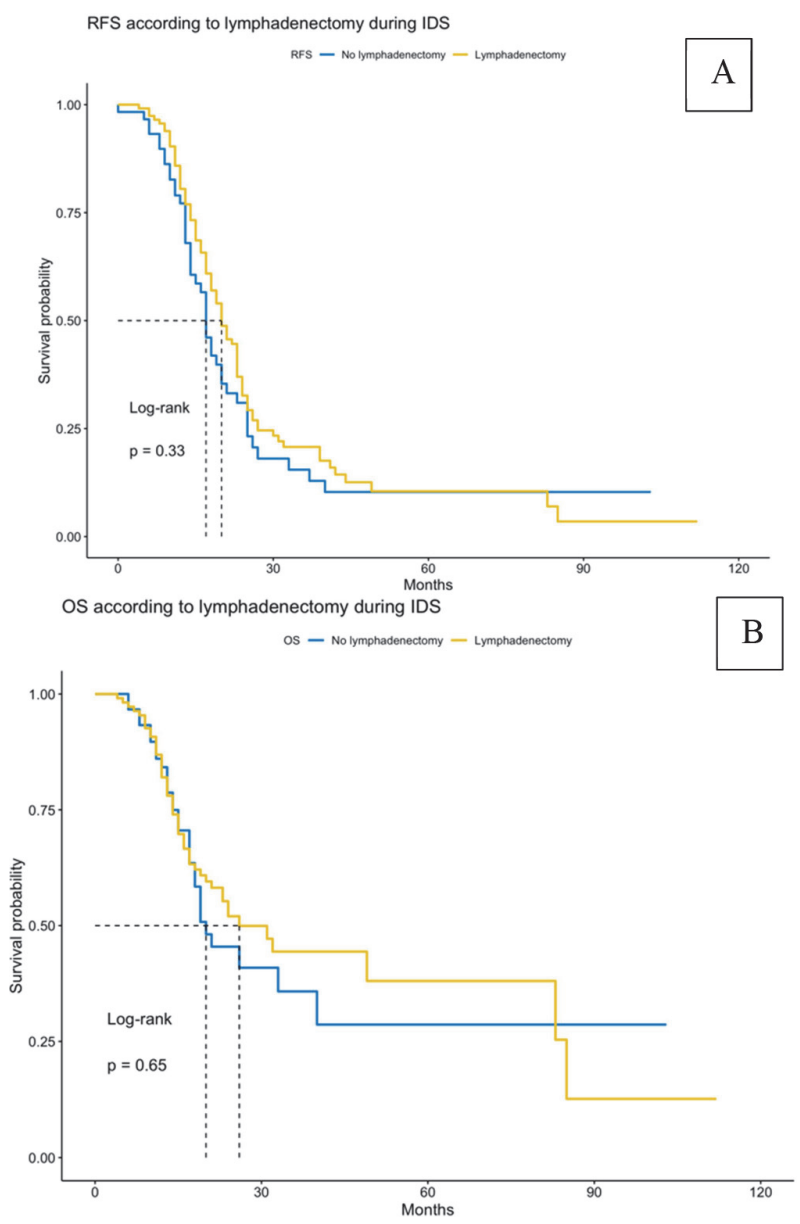

Abstract 240 Figure 1 Patients with a stage IIB-IV high grade serous ovarian cancer who underwent neo-adjuvant chemotherapy followed by interval debulking surgery either with or without systematic lymphadenectomy (LND)

A. Recurrence free survival (RFS) of patients with a stage IIB-IV ovarian cancer who underwent neo-adjuvant chemotherapy followed by interval debulking surgery $(p=0.3)$

B. Overall survival (OS) of patients with a stage IIB-IV ovarian cancer who underwent neo-adjuvant chemotherapy followed by interval debulking surgery $(p=0.6)$ 